\title{
Artizon Cloud: A Multidatabase System Architecture for an Art Museum
}

\author{
Naoki ISHIBASHI a,1 \\ ${ }^{a}$ Faculty of Data Science, Musashino University
}

\begin{abstract}
.
For dynamically integrating professional knowledge of curators, a multidatabase system architecture for an art museum, Artizon Cloud is proposed. A location based data provision is defined in the architecture for visitors. A system and applications are implemented and provided in an actual museum, and heterogeneous archives that were independently implemented as databases with Web UIs are dynamically extracted, integrated, and staged in visitors' devices.
\end{abstract}

Keywords. multidatabase systems, museum systems, multimedia databases

\section{Introduction}

By combining art works and advanced knowledge of curators, art museums provide intellectual and emotional experiences to all visitors. Many kinds of devices have been tested to provide the knowledge to users, but a framework to dynamically stage intellectual properties of curators to visitors is not proposed.

In the past years, museums started to develop many kinds of digital archives for photographs, evidential documents, or exhibitions. Therefore, intellectual properties of artists and curators are now becoming digital archives especially photographs of art works[1,2,3]. Moreover, researches beyond archiving photographs have been proposed to store and share knowledge such as knowledge co-creation on social networking paradigm to maintain a cultural portal[4], archive for museum layouts with visitor behavior analysis[5] and archives for traditional culture and directing new experiences using digital technologies[6].

A multidatabase system[7,8,9] that dynamically integrates the digital archives built by curators to provide services is desired especially for museums which do not employ engineers. By dynamically integrating the archives that represent knowledge of curators and/or art work themselves, the knowledge could automatically provide newer intellectual or entertaining services. For integrating legacy databases, managing heterogeneity is crucial to establish multidatabase systems[10], and a metadatabase approach $[11,12,13,14]$ is observed to fit with museums since following reasons: 1) metadata domain to share among archives is definable, 2) semantic computing is required for many kinds of art data.

\footnotetext{
${ }^{1}$ Corresponding Author: Naoki Ishibashi, Faculty of Data Science, Musashino University, 3-3-3, Ariake, Kotoku, Tokyo, Japan; E-mail:n-ishi@musashino-u.ac.jp.
} 


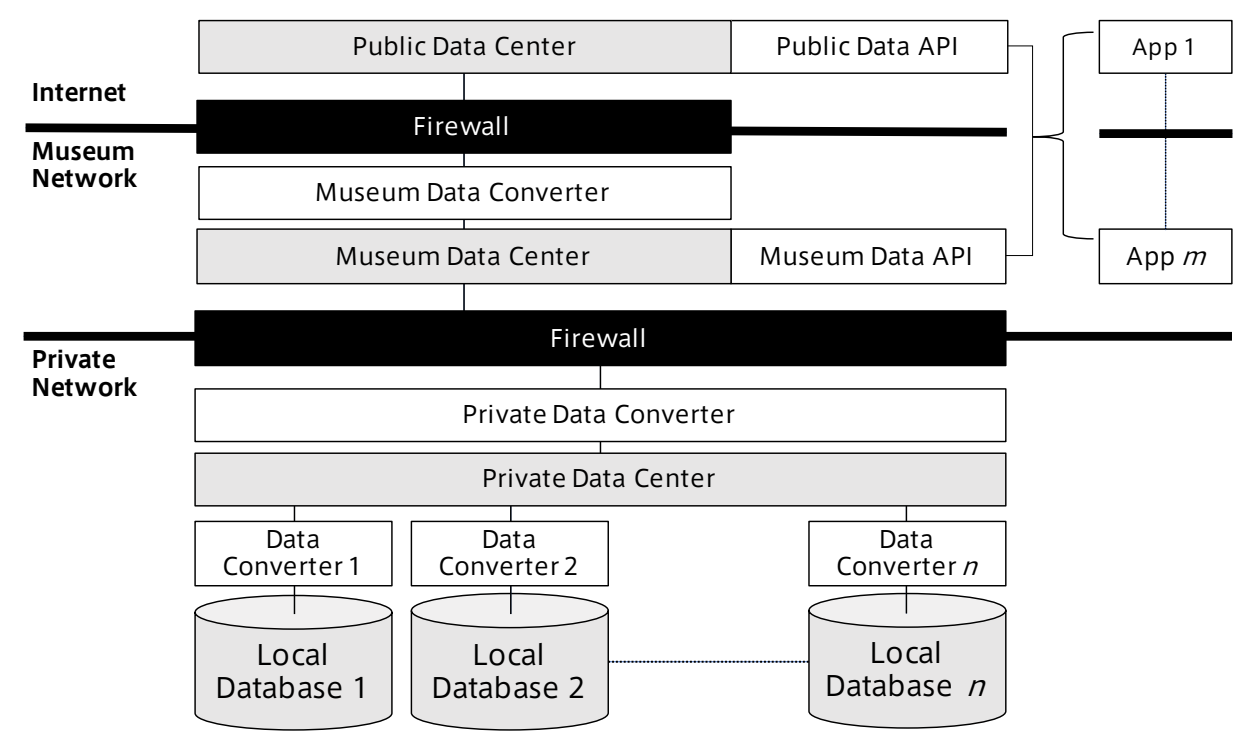

Figure 1. A System Architecture of Artizon Cloud

In this paper, a multidatabase system architecture, Artizon Cloud, for a museum is proposed. For an art collection, Curators edit data in archives that are managed in a local network, Artizon Cloud dynamically integrates the archives and provide appropriate data to either museum visitors or WWW users.

\section{A Multidatbase System Architecture}

By defining three distinct areas to provide information, Artizon Cloud is designed as Fig. 1 such as Private Network, Museum Network, and Internet.

Curators register and manage art collection data in web-based data archives in a local network with private IP addresses. This Private Network is accessible only by staff members, and the archives are privately maintained. Each archive uses distinct DBMS, and schemas are independently designed and implemented. Data in these archives are converted to Private Data Center, and Private Data Center integrates various data types.

Private Data Center sends a data set that includes information for museum visitors and WWW users through Private Data Converter to Museum Data Center as Fig. 2. Therefore museum visitors can access a lot of art information more than WWW users, such as high-resolution graphic, restricted audio or video data, as well as all information available on WWW. It means a museum with Artizon Cloud can provide massive amount of knowledge automatically converted from curators' daily work to the museum visitors.

Museum Data Center sends a set of data for WWW users through Museum Data Converter to Public Data Center, and Public Data Center is a back end to implement WWW applications as well as smartphone applications. Once more, the daily works of the curators are converted to Public Data Center, so Artizon Cloud reduces tasks to publish various data to WWW and it also provides infrastructure to develop new museum applications. 


\begin{tabular}{|c|c|c|c|c|}
\hline \multirow[t]{3}{*}{ Public } & data 1 & data2 & data 3 & area \\
\hline & 1 & a & AAA & public \\
\hline & 2 & b & BBB & public \\
\hline \multirow[t]{5}{*}{ Museum } & data 1 & data2 & data 3 & area \\
\hline & 1 & a & AAA & public \\
\hline & 2 & $b$ & BBB & public \\
\hline & 3 & c & $\mathrm{CCC}$ & museum \\
\hline & 4 & d & DDD & museum \\
\hline \multirow[t]{6}{*}{ Private } & data 1 & data 2 & data3 & area \\
\hline & 1 & a & AAA & public \\
\hline & 2 & b & BBB & public \\
\hline & 3 & c & $\mathrm{CCC}$ & museum \\
\hline & 4 & $d$ & DDD & museum \\
\hline & 5 & e & EEE & private \\
\hline
\end{tabular}

Figure 2. A Data Flow in Artizon Cloud

Many kinds of applications are assumed of Artizon Cloud as shown in Fig. 3. Museum Data Center can provide applications on devices of a group of visitors and applications on museum devices. Furthermore, this architecture could provide capability to quickly develop a museum application in an actual museum, so the art museum could become an experimental space for open data innovation $[15,16]$.

\section{A Staging Environment}

As a reconstruction of Bridgestone Museum of Art that was established in 1952, Artizon Museum[17] started in January 2020 in Kyobashi, Tokyo. The scope of the museum extends from antique art works, modern Japanese Western-style paintings, the Impressionists, 20th century art, to contemporary art, and approximately 3,000 art works are managed in a collection. Three floors, approximately $2,100 \mathrm{~m}^{2}$ in total, are available as galleries, and Wi-Fi is provided to all floors for visitors in a local network with private IP addresses.

\section{An Implementation of Artizon Cloud}

Artizon Cloud was actually implemented for Artizon Museum, and started to provide services since January 2020. At this point, five archives are connected to Artizon Cloud 


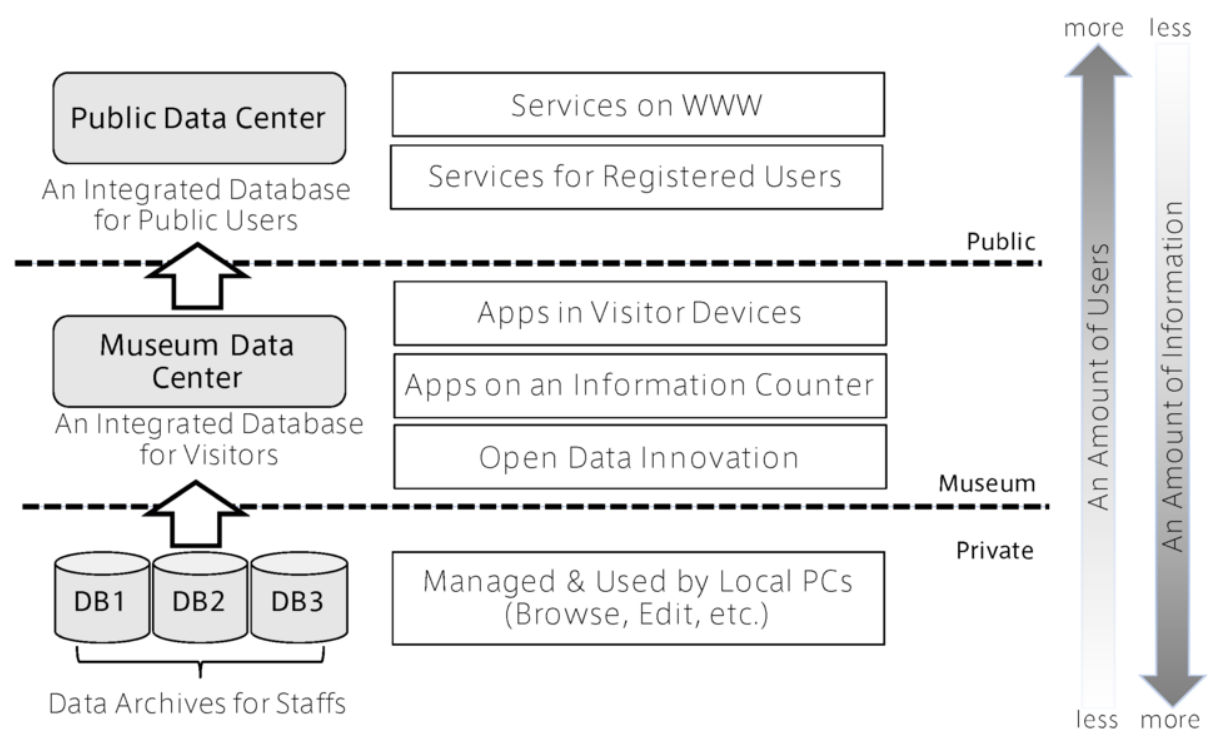

Figure 3. Service Examples of Artizon Cloud

as shown in Fig. 4, and these archives are independently implemented by different organizations. However, only exception is that metadata extracted from Artize are shared with other systems.

\subsection{Archives}

Following archives are independently implemented, and all these archives are WebDB systems.

\subsubsection{Artize}

Artize is a collection management system, and a commercial software developed by Nissha Co., Ltd. customized for Artizon Museum. Curators of the museum register data of art works in the collection, and manage a set of relative data, such as names of artists, names of art works, histories of art works, repair records, exhibition logs, photographs, references, etc. Data in Artize include most of detailed information for all art works of the collection, and the data are daily maintained by the curators. The most recent data could be discovered from Artize, and a set of metadata is automatically extracted from Artize as follows: data of 1) art works, 2) artists, and 3) exhibitions. Therefore, Artize is the most significant archive for Artizon Cloud, because it provides these 3 metadata to Private Data Center as well as to other archives.

\subsection{2. iDash}

iDash is an evidential document archive developed by Governance Design Laboratory, Inc., and it manages evidential documents of art works or artists, repair histories of art works, and publications of the museum. 


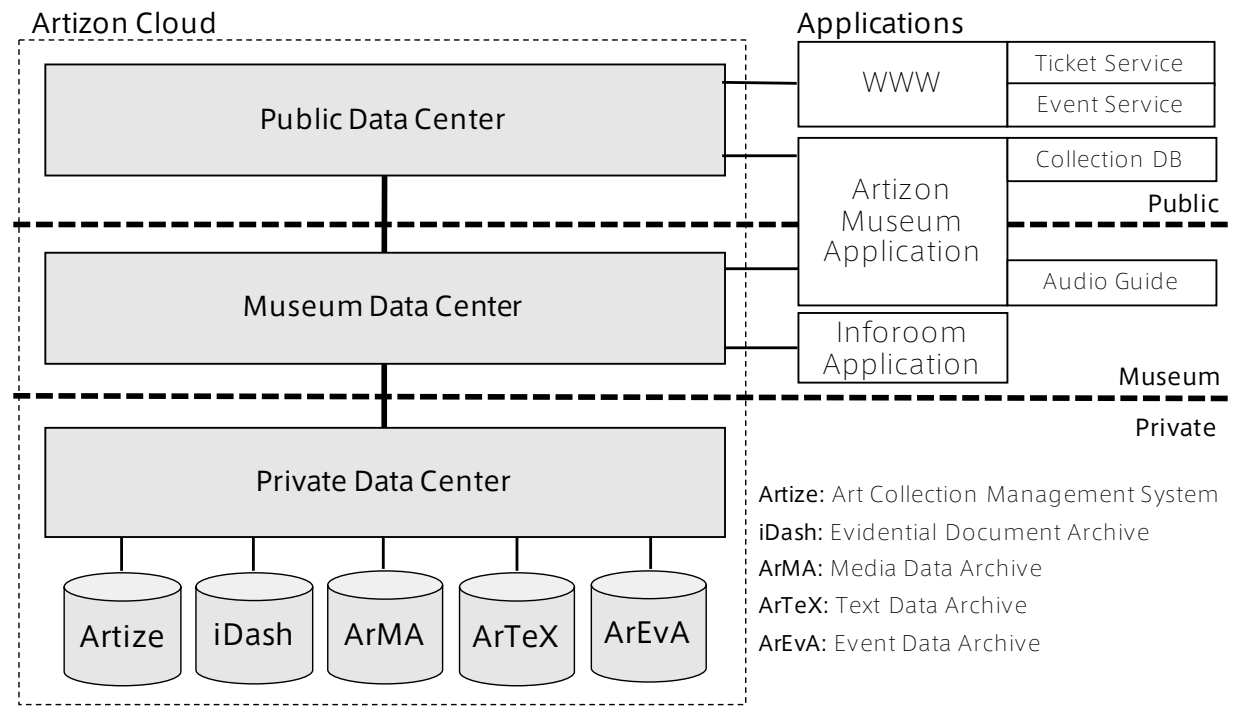

Figure 4. An Implementation of Artizon Cloud

\subsection{3. $\operatorname{ArMA}$}

ArMA is a media data archive of art works, artists, and exhibitions to manage graphic, audio, or video data, and ArMA was implemented by aery Co. ArMA was initially planned to manage high-resolution photographs as evidences to repair art works, and expanded its role to manage media data such as movies of each exhibition, and audio files for an audio guide in the museum, etc.

\subsection{4. $\operatorname{ArTeX}$}

ArTeX was implemented by Newphoria Corporation to manage texts written by the curators. The curators of Artizon Museum used to write various text documents using MS Word, and stored data in each PC. To realize unified storage of texts, ArTeX receives MS Word files and extracts texts into DBMS. Any language could be stored using UTF-8, but English, Japanese, Chinese and Korean languages are recently stored.

\subsection{5. $\operatorname{ArEvA}$}

ArEvA was implemented by Dai Nippon Printing Co., Ltd. to store event data such as exhibitions, lectures, or symposiums. It was designed to store a history of the museum including posters of each exhibition.

\subsection{Private Data Center}

The sets of data from these archives are automatically extracted and converted to Private Data Center, that we call MetaDB. MetaDB currently has functions as follows:

- A function to extract, convert, and store metadata for the art works, the artists, and the exhibitions in an ORDB and a file system from Artize 
- A function to provide the metadata to the archives via Web API

- Functions to edit the metadata such as to set an appropriate zone, private, museum, or public, to each datum via Web UI

- Functions to store the data of each archive in the ORDB and the file system

- Functions to edit the data of the archives such as private, museum, or public via Web UI

- Functions to edit copyrights of the graphics such as the art works, or the posters via Web UI

- A function to convert and transfer the metadata to Museum Data Center

- Functions to convert and transfer the data of the archives to Museum Data Center

\subsection{Museum Data Center}

The set of data from MetaDB is automatically received and converted to Museum Data Center, that we call $D M Z D B$. DMZDB currently has functions as follows:

- A function to store metadata for the art works, the artists, and the exhibitions in an ORDB and a file system

- Functions to store the data of the archives in the ORDB and the file system

- Functions to provide a data set of the art works via Web API in the museum

- Functions to provide a data set of the artists via Web API in the museum

- Functions to provide a data set of the exhibitions via Web API in the museum

- Functions to provide a data set of the publications via Web API in the museum

- A function to convert and transfer a data set of the art works to Public Data Center

- A function to convert and transfer a data set of the artists to Public Data Center

- A function to convert and transfer a data set of the exhibitions to Public Data Center

\subsection{Public Data Center}

Public Data Center is implemented in EC2 and S3 of Amazon Web Services,AWS, and it currently has functions as follows:

- Functions to provide a data set of the artists via Web API

- Functions to provide a data set of the exhibitions via Web API

- Functions to provide a data set of the publications via Web API

\section{An Application of Artizon Cloud}

As a first application of Artizon Cloud, Artizon Museum App. was released in late 2019[18], and sample screens of the application are presented in Fig. 5.

When a visitor arrives at the museum, their devices are recommended to connect to the $\mathrm{Wi}-\mathrm{Fi}$ in the museum. Then, these devices are capable to access all information provided from DMZDB such as audio guide data of an actor(Fig. 6). In galleries, beacon devices are embedded to specific art works that have audio files to guide, and these devices detect a location of an user to switch a screen of the application.

Since the grand opening of Artizon Museum in January 2020, 108,770 people have arrived at Artizon Museum, 9,311 downloads were observed for the iOS application, 

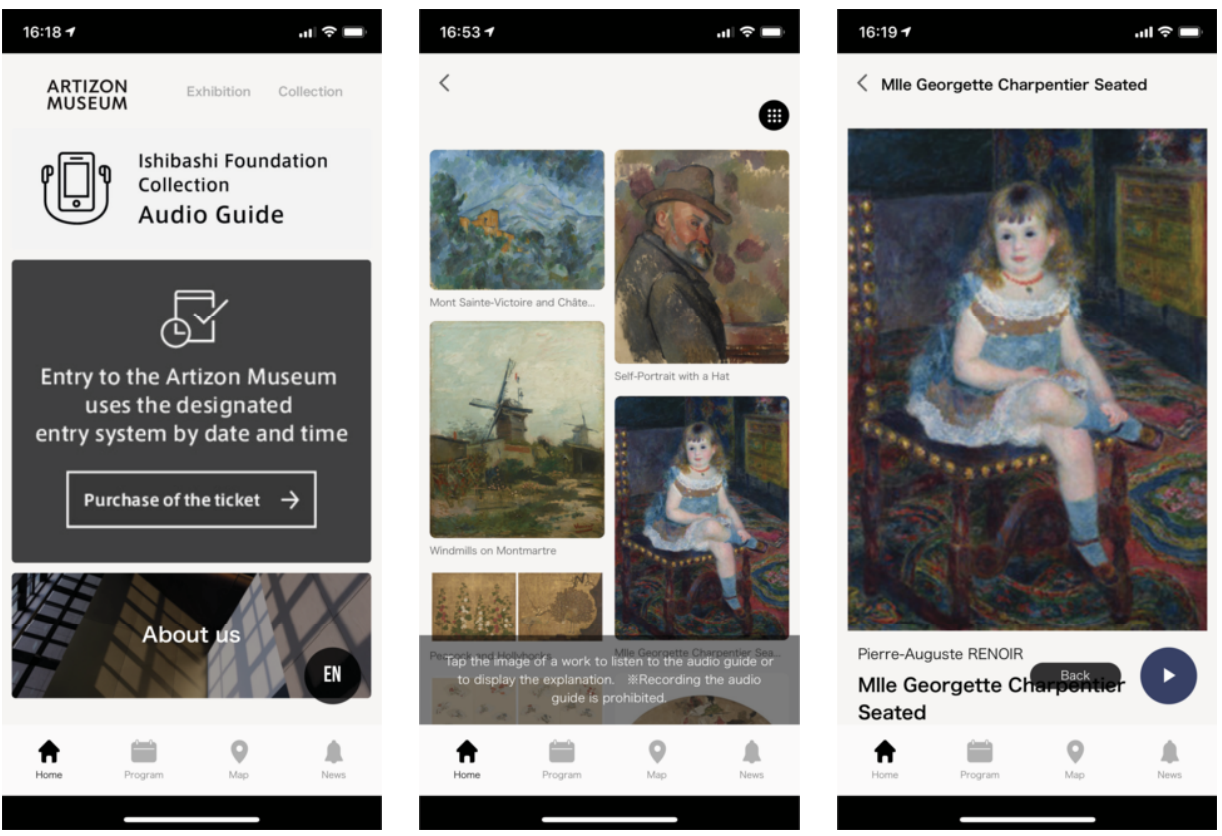

Figure 5. Sample Screens of Artizon Museum App.

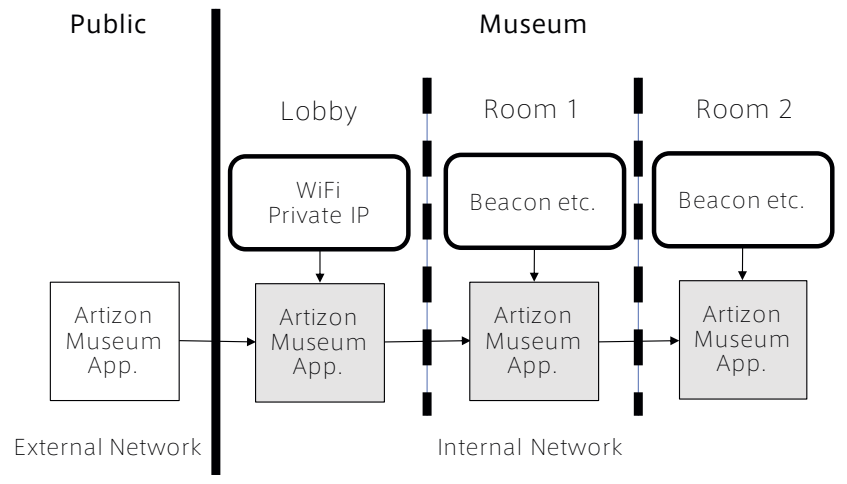

Figure 6. Zone Transitions of Artizon Museum App.

and 4,983 downloads were observed for the Android Application until end of 2020. The numbers of visitors using the application are still small, though many and very positive feedback were observed on various SNSs.

Since Artizon Cloud and the application, Artizon Museum stopped lending devices to visitors, and is encouraging people to bring their own devices and earphones to the museum. Artizon Museum has a strong will to produce intellectual and emotional experiences by providing massive amount of data. 


\section{Conclusion}

A multidatabase system architecture for an art museum is proposed. Artizon Cloud dynamically integrates independent archives of the art collection, and provide data according to user's location such as in an office, an museum, and elsewhere. The system is implemented and the services are provided to the visitors of the actual museum, Artizon Museum, and iOS and Android applications are currently available.

By applying the implemented system, advanced knowledge of the curators as well as art works themselves are dynamically extracted, integrated and staged to the actual smartphone applications of visitors, the visitors can access audio guides, videos and collection data by plugging in their earphones.

As future works, we are discussing to design methodology of dynamic curation in a cyberspace, an entertainment application for children, and we are also planning to encourage other art museums to join our challenge.

\section{Acknowledgement}

Without the vision for the new art museum of Hiroshi Ishibashi, Chairman of Ishibashi Foundation, this project would not even exist. I would like to express my utmost gratitude to him for this vision and his thirst for the future of art museums.

I would like to express my sincere gratitude to Taiji Nishijima, Michiko Kasahara, Tadashi Urami, and Kazunori Yamauchi of Ishibashi Foundation for their great cooperation and support throughout the project period.

Shunsuke Naotsuka, Shoji Kometani, and Tomohiro Kawasaki of Ishibashi Foundation were always my colleagues in the project for years. Without their help, there is no doubt that this project would not have reached its goal.

The members of the curatorial department and the public relations department of Ishibashi Foundation have also shared many meetings and discussions with us. Without the opinions and cooperation of the people on the ground, we would not be able to create a core system for the organization. I would like to express my gratitude to them for their continuous cooperation.

I would also like to express my gratitude to all the organizations that have contributed to the overall system. I would like to express my sincere gratitude to them for their dedication and continuous efforts.

Finally, I would like to express my highest appreciation to Professor Yasushi Kiyoki of Keio University. I am sure that our experience together at Keio University was the initial inspiration for this research. I would like to take this opportunity to thank him.

\section{References}

[1] The Metropolitan Museum of Art: The Met Collection, available via WWW, https://www. metmuseum.org/art/collection (2021)

[2] Musée du Louvre: Atlas database of exhibits, available via WWW, http://cartelen.louvre.fr/ (2021).

[3] Paris Musées: Les collections en ligne des musées de la Ville de Paris, available via WWW, https: //www.parismuseescollections.paris.fr/ (2021). 
[4] Sornlertlamvanich, V. and Charoenporn, T.: "Cultural Knowledge Co-Creation on Social Networking Paradigm," Proceedings of The Second International Conference on Culture and Computing, Kyoto University, Kyoto, Japan 2011).

[5] Kovavisaruch, L., Sanpechuda, T., Chinda, K., Kamolvej, P. and Sornlertlamvanich, V.: "Museum Layout Evaluation based on Visitor Statistical History," Asian Journal of Applied Sciences, Vol.5, No.3, pp.615-622 (2017).

[6] Digitized Thailand: White Paper, NECTEC, available via WWW, https://www.facebook.com/ digitizedthailand (2009).

[7] Batini, C., Lenzerini, M. and Navathe, S.B.: "A comparative analysis of methodologies for database schema integration", ACM Computing Surveys, Vol.18, No.4, pp.324-364 (1986).

[8] Litwin, W., Mark, L. and Roussopoulos, N.: "Interoperability of Multiple Autonomous Databases", ACM Comp. Surveys, Vol.22, No.3, pp.267-293 (1990).

[9] Sheth, A.P. and Larson, J.A.: "Federated database systems for managing distributed, heterogeneous, and autonomous databases," ACM Computing Surveys, Vol.22, No.3, Special issue on heterogeneous databases, pp.183-236 (1990).

[10] Zhang, J.: "Classifying approaches to semantic heterogeneity in multidatabase systems," Proceedings of the 1992 conference of the Centre for Advanced Studies on Collaborative research - Volume 2, pp.153173 (1992).

[11] Kitagawa, T. and Kiyoki, Y.: "The mathematical model of meaning and its application to multidatabase systems," Proc. 3rd IEEE Int. Workshop on Research Issues on Data Engineering: Interoperability in Multidatabase Systems, p.130-135 (1993).

[12] Kiyoki, Y. and Kitagawa, T.: "A metadatabase system supporting interoperability in multidatabases", Information Modeling and Knowledge Bases, Vol.5, pp.287-298 (1993).

[13] Kiyoki, Y., Kitagawa, T. and Hitomi, Y.: "A fundamental framework for realizing semantic interoperability in a multidatabase environment", Journal of Integrated Computer-Aided Engineering, Vol.2, No.1, pp.3-20 (1995).

[14] Kiyoki, Y., Hosokawa, Y. and Ishibashi, N.: "A Metadatabase System Architecture for Integrating Heterogeneous Databases with Temporal and Spatial Operations," Advanced Database Research and Development Series Vol. 10, Advances in Multimedia and Databases for the New Century, A Swiss/Japanese Perspective, pp.158-165, World Scientific Publishing (1999).

[15] Janssen, M., Charalabidis, Y. and Zuiderwijk, A.: "Benefits, Adoption Barriers and Myths of Open Data and Open Government," Information Systems Management, pp.258-268. (2012)

[16] Zuiderwijk, A., Janssen, M., and Davis, C.: "Innovation with Open Data: Essential Elements of Open Data Ecosystems", Information Polity, pp. 17-33. (2014)

[17] Artizon Museum: Artizon Museum, available via WWW, https://www.artizon.museum/en/ (2021).

[18] Artizon Museum: Artizon Museum Official App, available via WWW, https://www.artizon. museum/en/user-guide/application/ (2021). 